\title{
Fracture of Crystalline Germanium during Electrochemical
}

\section{Lithium Insertion}

Seok Woo Lee ${ }^{1}$, Ill Ryu ${ }^{2}$, William D. Nix ${ }^{2}$, and Yi Cui ${ }^{2,3^{*}}$

${ }^{I}$ Geballe Laboratory for Advanced Materials, Stanford University, Stanford, CA, 94305, USA.

${ }^{2}$ Department of Materials Science and Engineering, Stanford University, Stanford, CA, 94305,

USA. ${ }^{3}$ Stanford Institute for Materials and Energy Sciences, SLAC National Accelerator Laboratory, 2575 Sand Hill Road, Menlo Park, CA 94025, USA.

"Corresponding author: Y.C. email: yicui@stanford.edu. Adress: 476 Lomita mall, McCullough building 343, Stanford, CA 94305, USA. Phone: +1-650-723-4613

Germanium is one of the promising alloying anode ( $\mathrm{Si}, \mathrm{Ge}, \mathrm{Sn}$ ) materials for high capacity lithium ion batteries. Since it is isostructural with crystalline silicon, crystalline Ge is expected to show intriguing lithiation-induced phenomena similar to $\mathrm{Si}$, such as anomalous volume expansion and fracture. Here, we present the study of lithiation of Ge micropillars, and we compare the findings to silicon pillar lithiation. The critical pillar diameter $\sim 1.2 \mu \mathrm{m}$ associated with lithiation-induced fracture of $<111>$ Ge pillars, determined through our statistical investigation, is much greater than the critical dimension for fracture of <111> silicon pillars $(\sim 300 \mathrm{~nm})$. This larger critical size for lithiation-induced fracture of Ge likely arises from lower tensile stress concentrations at the surface due to the more inherently isotropic expansion that Ge undergoes during lithiation. Upon lithiation, Ge displays only slight anisotropy in its volume expansion, with the $\langle 110\rangle$ directions exhibiting radial 
expansion that is only 1.1 times larger than that along <111> directions. Despite its relatively weak anisotropy in volume expansion, however, Ge pillars above the critical dimension still show anisotropic fracture, with favored fracture sites residing between the $<110>$ directions on the pillar sidewall, similar to $\mathrm{Si}$. We believe that this study provides better understanding of lithiation of Ge for designing high-density anode for Li-ion batteries.

Keywords: germanium, Li-ion batteries, anode, anisotropic expansion, fracture

Alloying anode materials are considered promising anode electrodes for Li-ion batteries because of their high specific capacity made possible by reformation of chemical bonds and severe structural transformation ${ }^{1-3}$. However, alloying anodes usually suffer from rapid capacity decay due to mechanical fracture and instability of the solid electrolyte interphase (SEI) caused by large volume changes during repeated electrochemical lithium insertion and extraction ${ }^{4,5}$. In light of such shortcomings, a great deal of fundamental research into lithiation/delithiationinduced volume expansion/contraction and consequent fracture of such anode materials has been carried out ${ }^{6,7}$. Especially, silicon has been extensively studied due to its highest specific capacity among alloy anodes and its relative abundance ${ }^{8-12}$. Contradicting the former thought that $\mathrm{Si}$ lithiates via isotropic lithium $(\mathrm{Li})$ reaction and diffusion, we recently showed that $\mathrm{Si}$ undergoes anisotropic volume expansion, with expansion most significant along $\langle 110\rangle$ directions ${ }^{13,14}$. These were later confirmed by others ${ }^{15,16}$. Furthermore, we and others have found anomalous fracture behavior of crystalline Si nanostructures upon lithiation; fracture has been seen to occur preferentially between neighboring $<110>$ lateral directions due to the concentrated near-surface 
tensile hoop stresses that develop between these rapidly expanding directions ${ }^{17-19}$. Fracture promotion due to anisotropic expansion produces a relatively small critical pillar diameter for lithiation-induced fracture of crystalline Si pillars. Specifically, such Si pillars are found to fracture during lithiation when the initial pillar diameters are greater than as little as $\sim 300 \mathrm{~nm}^{17}$.

Although Ge is recognized as another promising anode material with a large theoretical specific capacity $(1600 \mathrm{mAh} / \mathrm{g})$, understanding its lithiation behavior is necessary for designing highdensity anode electrode for $\mathrm{Li}$-ion batteries ${ }^{20-23}$. Here, we utilize Ge nano/micro pillar structures with various axial crystal orientations for systematic study of the size effect for mechanical fracture as well as the volume expansion behavior of crystalline Ge upon electrochemical lithiation.

The procedures utilized for lithiation of single crystalline Ge pillars are similar to those of previous Si pillar lithiation research ${ }^{13,24,25}$. Ge micropillars were fabricated by dry etching single crystalline Ge wafer of three crystal orientations $(\langle 100\rangle,\langle 110\rangle,\langle 111\rangle)$ that ultimately define the etched pillars' axial orientations. Prior to etching, drop-cast Polystyrene (PS) microspheres with diameters of 1 and $2 \mu \mathrm{m}$ were first distributed on the wafer to be an etch mask and define the diameter of the etched pillar. The diameters of the etched pillars varied from $0.5 \mu \mathrm{m}$ to 1.7 $\mu \mathrm{m}$, and the heights varied from $4 \mu \mathrm{m}$ and $5 \mu \mathrm{m}$. After dry etching of the pillar, PS microspheres were removed by gentle sonication, and the sample were then cut to pieces with area of $\sim 25 \mathrm{~mm}^{2}$ that could be used as the working electrode in half-cell with Li-foil counter electrode. Next, the Ge micropillar samples were lithiated by sweeping the applied voltage at a specified rate until reaching a cut-off voltage of $10 \mathrm{mV}$ vs. $\mathrm{Li} / \mathrm{Li}^{+}$. The cut-off voltage was held for 10 hours as lithiation was allowed to proceed. The sweep rates were $0.1 \mathrm{mV} / \mathrm{s}$ and $1 \mathrm{mV} / \mathrm{s}$. After lithiation of 
Ge micropillars, the samples were washed in acetonitrile in an Ar filled glove box and transferred to a scanning electron microscope (SEM) chamber for observation of pillar morphologies.
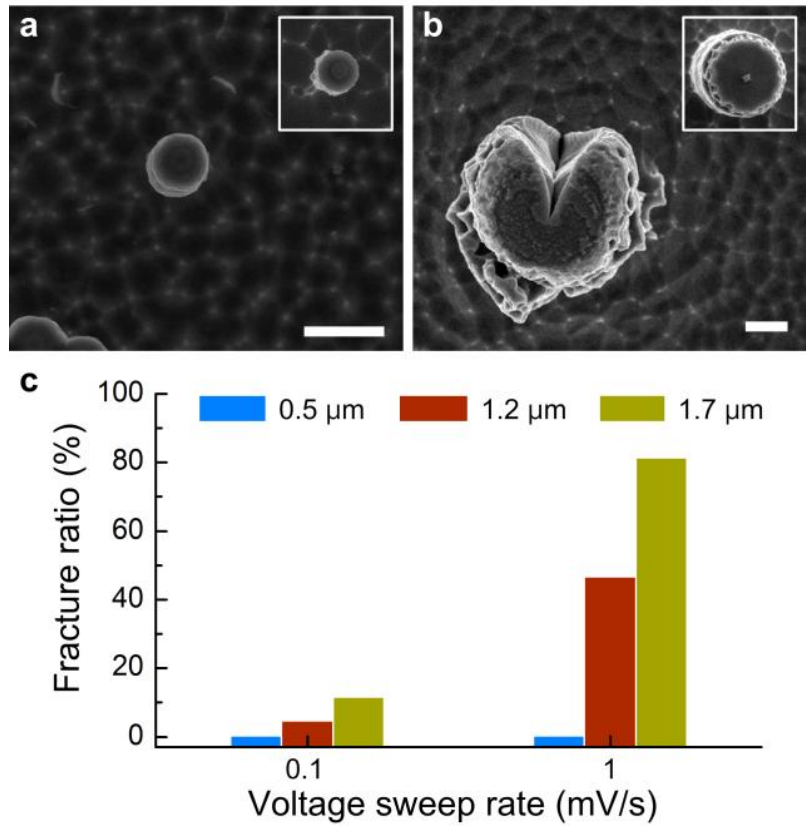

Figure 1 | Size effect for mechanical fracture of crystalline Ge pillar upon electrochemical

lithiation. a, Top-down-view SEM image of an unfractured, lithiated $<111\rangle$ Ge pillar of $0.5 \mu \mathrm{m}$ initial diameter. The inset is an SEM image of a pristine Ge pillar. b, Top-down-view SEM image of a fractured, lithiated $<111>$ Ge pillar of $1.7 \mu \mathrm{m}$ initial diameter. The inset is an SEM image of a pristine Ge pillar. All scale bars are $1 \mu \mathrm{m}$. The voltage sweep rates of both lithiated pillars are $1 \mathrm{mV} / \mathrm{s}$. c, Column chart of the fracture ratio for $<111>$ Ge pillars of various initial diameters and two different lithiation voltage sweep rates. 
Fig. 1 shows the size effect of mechanical fracture of $\langle 111\rangle$ Ge pillars upon lithiation. Ge pillars with $0.5 \mu \mathrm{m}$ diameters expand in a nearly isotropic manner and without observed fracture as shown in Fig. 1a while Si pillars larger than about $300 \mathrm{~nm}$ in diameter exhibit significant fracture $^{17}$. It agrees with the previous in-situ TEM study of fracture resistance of crystalline Ge particles upon lithiation ${ }^{26}$. Despite their robustness, Ge pillars also exhibit a size effect of fracture upon lithiation, as Si does. Fig. 1b clearly shows the fracture of Ge pillars upon lithiation when their initial diameter increases to $1.7 \mu \mathrm{m}$. To investigate the size effect and reaction rate effect for fracture, a statistical study was used to measure the fracture ratio for various pillar diameters with the reaction rate controlled by the voltage sweep rate as shown in Fig. 1c. The examined diameters of the Ge pillars were $0.5 \mu \mathrm{m}, 1.2 \mu \mathrm{m}$, and $1.7 \mu \mathrm{m}$ and voltage sweep rates were $0.1 \mathrm{mV} / \mathrm{s}$ and $1 \mathrm{mV} / \mathrm{s}$. When the initial diameter of Ge pillar is $0.5 \mu \mathrm{m}$, the fracture is not found regardless of the voltage sweep rate (blue column). When the initial pillar diameter is increased to $1.2 \mu \mathrm{m}$ (red column) and $1.7 \mu \mathrm{m}$ (green column), the lithiated pillars exhibit some fracture and the fracture ratios are $4.3 \%$ and $11.3 \%$ for voltage sweep rates of $0.1 \mathrm{mV} / \mathrm{s}$, respectively. For the faster voltage sweep rate $(1 \mathrm{mV} / \mathrm{s})$, the lithiated pillars with initial diameters of $1.2 \mu \mathrm{m}$ and $1.7 \mu \mathrm{m}$ show a significant increase of fracture ratio to $46.4 \%$ and $81.1 \%$, respectively. 

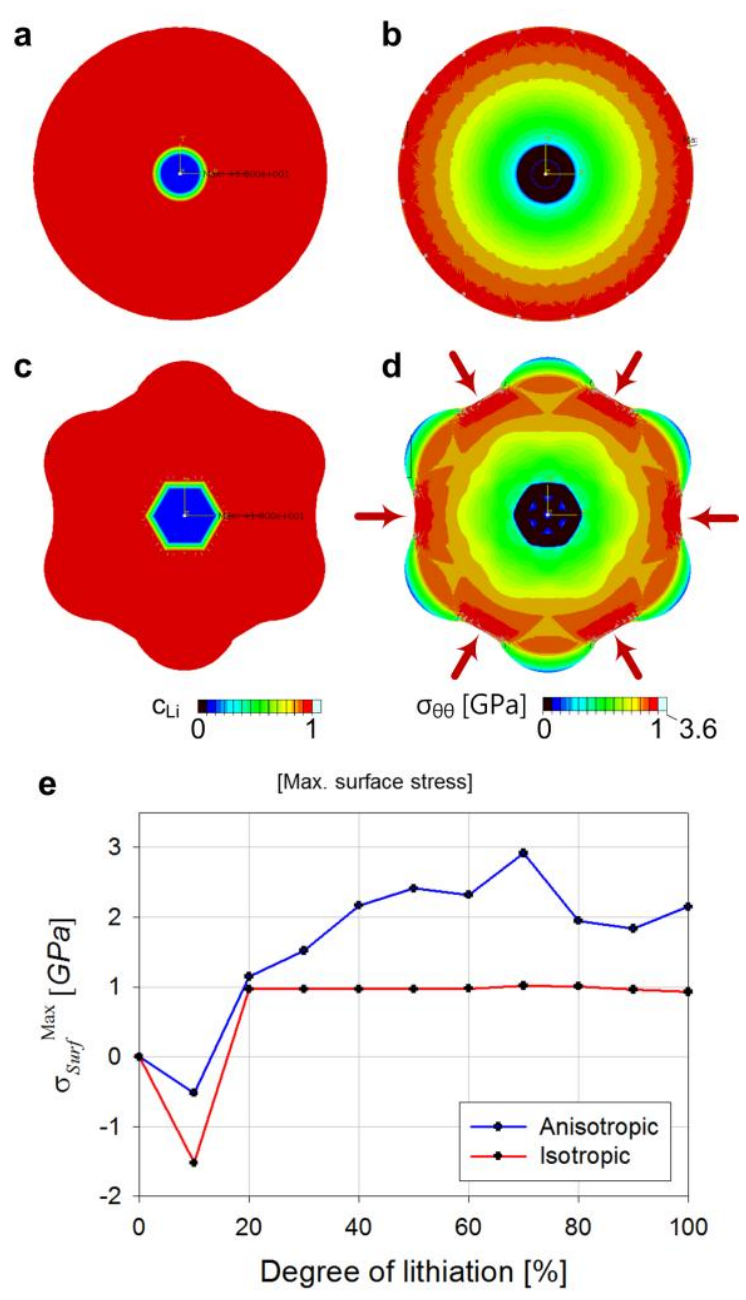

Figure 2 | Finite element analysis of hoop stress profile of crystalline Ge pillar upon

lithiation. a, Li concentration profile of a modeled Ge pillar with circular moving interface at $80 \%$ lithiation for isotropic volume expansion. b, Hoop stress profile of a modeled Ge pillar with an assumed circular lithiation front (isotropic volume expansion) shown at $80 \%$ lithiation. c, Li concentration profile of a modeled $<111>$ Ge pillar with an assumed hexagonal moving interface at $80 \%$ lithiation for anisotropic volume expansion. d, Hoop stress profile of a modeled $<111>$ Ge pillar with an assumed hexagonal lithiation front (anisotropic volume expansion) shown at $80 \%$ lithiation. All simulation results are presented by illustrating an axial section of 
the pillar. e, Maximum hoop stress of a modeled Ge pillar plotted versus degree of lithiation for cases of isotropic and anisotropic volume expansion.

Finite-element-based lithiation simulations explain how isotropic expansion of lithiated Ge enhances fracture resistance (see Movie 1 and 2 in Supplementary Information). The initial diameter of the simulated Ge pillar is $1.0 \mu \mathrm{m}$. The artificial moving interface between crystalline Ge and $\mathrm{Li}_{\mathrm{x}} \mathrm{Ge}$ with $270 \%$ volume expansion is defined for lithiation of Ge pillar as in our previous study ${ }^{19}$. The mechanical properties of lithiated Ge utilized in formulating the finite element models were obtained from nanoindentation experiments into $\mathrm{Li}_{\mathrm{x}} \mathrm{Ge}$ samples (see Fig. S3 in Supplementary Information). The measured Young's modulus was $57.3 \mathrm{GPa}$, and the yield strength - taken as the hardness divided by the Tabor factor of 3-was measured to be $0.84 \mathrm{GPa}$. The case of isotropic expansion (Fig. 2a,b) is simulated by defining a circular interface between the lithiated and unlithiated material that remains circular as it travels radially inward. Fig. 2a shows the Li concentration $\left(\mathrm{c}_{\mathrm{Li}}\right)$ profile when the degree of lithiation is $80 \%$. The concentration of $\mathrm{Li}$ is zero at the crystalline $\mathrm{Ge}$ core and 1 at the $\mathrm{Li}_{\mathrm{x}} \mathrm{Ge}$ shell. There is a steep transition in the concentration profile from 0 to 1 across the interface. Fig. 2 b shows the hoop stress $\left(\sigma_{\theta \theta}\right)$ profile for isotropic expansion when the degree of lithiation is $80 \%$. It is evident that isotropic expansion induces uniformly distributed near-surface tensile hoop stresses bounded by the yield strength of lithiated Ge. For comparison, anisotropic expansion of $\langle 111\rangle$ Ge pillar is simulated by hexagonal shape of artificial moving interface. Fig. $2 \mathrm{c}$ shows Li concentration $\left(\mathrm{c}_{\mathrm{Li}}\right)$ profile with hexagonal interface when the degree of lithiation is $80 \%$. The corresponding hoop stress $\left(\sigma_{\theta \theta}\right)$ profile exhibits a concentration of tensile stress (red arrows) induced by anisotropic expansion as 
in our previous study (Fig. 2d) ${ }^{19}$. Fig. 2e compares the maximum hoop stress at the surface extracted from simulations plotted against degree of lithiation for both the cases of assumed isotropic and anisotropic expansion. Isotropic expansion induces evenly distributed tensile stress bounded by the yield strength of lithiated Ge while the anisotropic expansion gives rise to large tensile stresses that exceed the yield strength due to triaxiality at these stress concentration locations. Therefore, isotropic expansion behavior upon electrochemical lithiation could make crystalline Ge more resistant to fracture than crystalline $\mathrm{Si}$.
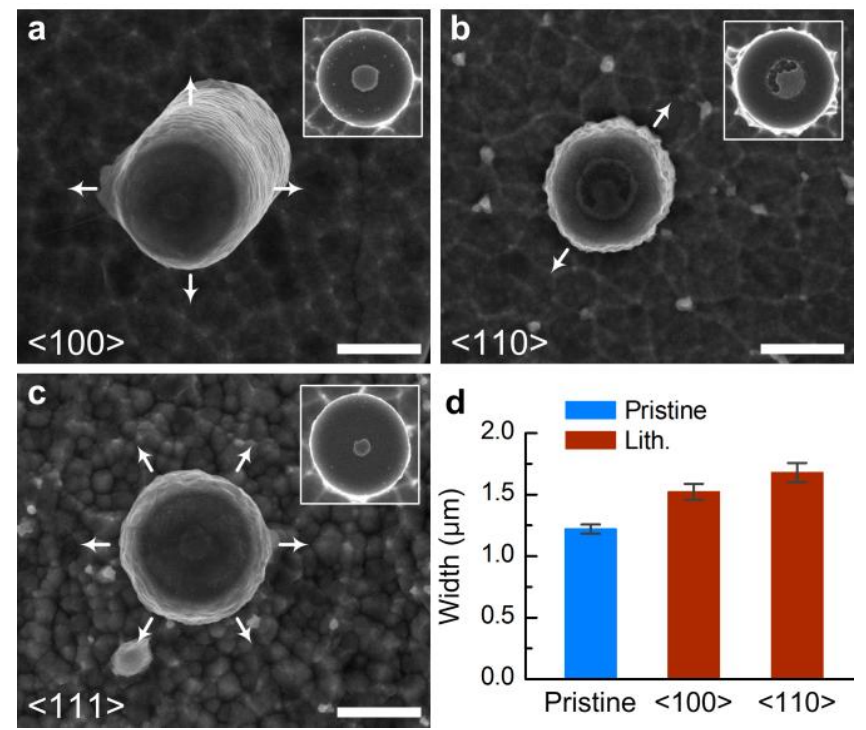

Figure 3 | Anisotropic expansion of crystalline Ge pillars with three kinds of crystal orientation, $\langle 100\rangle,\langle 110\rangle$, and $\langle 111\rangle$ upon electrochemical lithiation. a-c, SEM images from top-down view of Ge pillars of each crystal orientation after lithiation. White arrows indicate $\langle 110\rangle$ direction of preferential lithiation on the sidewall of the pillars. Insets are pristine pillars. All scale bars are $1 \mu \mathrm{m}$. d, Statistical data of lateral dimension of pristine and lithiated 
$\langle 110\rangle$ pillar along $\langle 110\rangle$ and $\langle 100\rangle$ direction. The voltage sweep rates of all lithiated pillars are $0.01 \mathrm{mV} / \mathrm{s}$.

Although crystalline Ge lithiates almost isotropically, the material still shows some minor preferential lithiation along the $\langle 110\rangle$ direction during electrochemical lithiation although its degree is much less than crystalline Si. Fig. 3 a-c are top-down views of SEM images of initially single crystalline Ge nanopillars of various axial orientation, <100>, <110>, <111> after lithiation. The insets are SEM images of pristine Ge pillars with same magnification for a comparison. The features at the center of the top surfaces of the pillars are artifacts of PS spheres utilized during the pillar etching-based fabrication process. Ge pillars with diameters of $1.2 \mu \mathrm{m}$ were lithiated by sweeping the voltage at rates of $0.1 \mathrm{mV} / \mathrm{s}$ and $1 \mathrm{mV}$ until the cutoff voltage is reached, whereupon the voltage is held constant for 10 hours. White arrows are indicating <110> directions on the sidewalls of the pillars on which Si nanopillar showed preferential volume expansion in our previous study ${ }^{13}$. Fig. 1a shows lithiated $\left.<100\right\rangle$ pillar that has four $<110>$ directions on the sidewall. After lithiation, the pillar expands nearly isotropically, although shallow grooves are shown between $\langle 110\rangle$ directions likely due to some preferred volume expansion occurred along $\langle 110\rangle$ directions. The $\langle 110\rangle$ Ge pillar shows more evident preferential lithiation along the $\langle 110\rangle$ direction and expands to a slightly elliptical shape as shown in Fig. $3 \mathrm{~b}$ since it has two $<110>$ directions on the sidewall. The $<111>$ Ge pillar expands to a circle without significant anisotropic expansion since it has six $\langle 110\rangle$ directions on the sidewalls (Fig. 3c). To clarify the anisotropy of the lithiated Ge pillars observed in SEM images, a statistical study was used to measure the dimensional changes of the pillars. Fig. $3 d$ shows statistical data of cross-sectional dimension changes of <110> lithiated Ge pillars along <100> and $\langle 110\rangle$ directions. About 30 pillars were measured to determine the average values 
(columns) and standard deviations (error bars). The average diameter of the pristine pillar is 1.22 $\mu \mathrm{m}$ and expands to $1.52 \mu \mathrm{m}$ along the $<100>$ direction and $1.68 \mu \mathrm{m}$ along the $<110>$ direction. It is clear that the $\langle 110\rangle$ direction has $10 \%$ more expansion than the $\langle 100\rangle$ direction. In comparison, Si nanopillars with a $<110>$ long axis show $400 \%$ more volume expansion along the $\langle 110\rangle$ direction compared to the $\langle 100\rangle$ direction ${ }^{13}$.
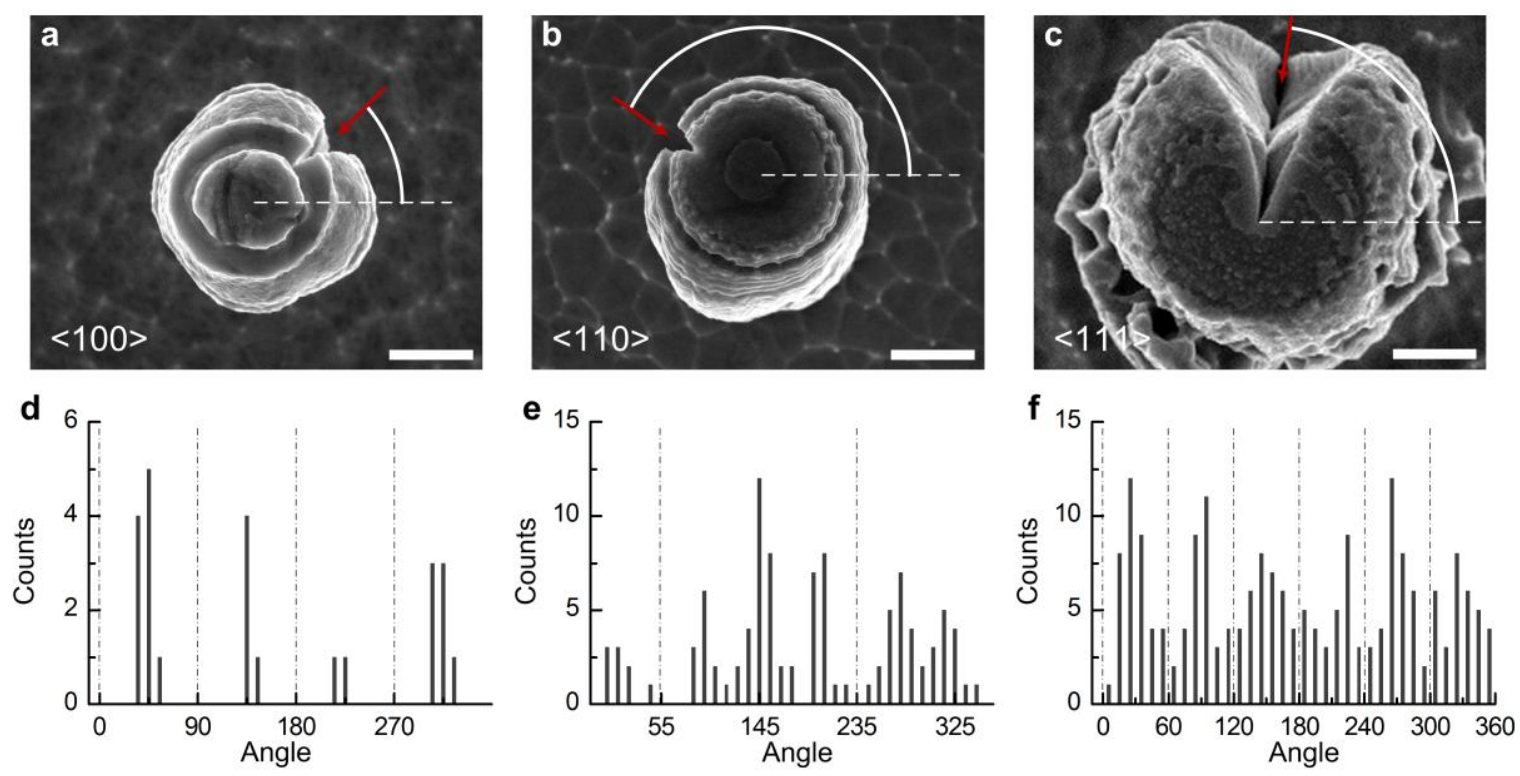

Figure 4 | Statistical study of fracture site of crystalline Ge pillar with three kind of crystal orientation, $\langle\mathbf{1 0 0}\rangle,\langle\mathbf{1 1 0}\rangle$, and $\langle\mathbf{1 1 1}\rangle$ upon lithiation. a-c, Top-down-view SEM images of Ge pillars of each axial orientation with fracture after lithiation. White dash lines are the reference point $\left(0^{\circ}\right)$. Red arrows indicate the location of cracks as an angle from the reference point. All scale bars are $1 \mu \mathrm{m}$. d-f, Population plot for the location of cracks in the pillars of each crystal orientation. Vertical dash lines indicate $\langle 110\rangle$ direction of the pillars of each crystal orientation. The voltage sweep rates of all lithiated pillars are $1 \mathrm{mV} / \mathrm{s}$. 
Our previous study showed that crystalline $\mathrm{Si}$ has favored fracture sites located between neighboring <110> directions as anisotropic expansion induces concentrated tensile hoop stresses during electrochemical lithiation. Although anisotropy in volume expansion of lithiated $\mathrm{Ge}$ is incomparably smaller than of lithiated $\mathrm{Si}$, it still influences the fracture behavior of $\mathrm{Ge}$ as it does for Si. Fig. 4a-c show lithiation-induced cracks in Ge pillars with initial $\langle 100\rangle,\langle 110\rangle$, and $<111>$ axial orientations, respectively. After lithiation, the pillars exhibit slight anisotropic expansion along the $\langle 110\rangle$ directions, and cracks are found between the $\langle 110\rangle$ directions. To clarify this tendency, a statistical study measured the location of the cracks in the lithiated Ge pillars of three axial orientations, $\langle 100\rangle,\langle 110\rangle$, and $\langle 111\rangle$ as shown in Fig. 4d-f, respectively. The diameters of pristine pillars of each orientation shown in the SEM images are all $1.7 \mu \mathrm{m}$. The location of the crack in the pillar is represented by the measured angular orientation of the crack from the horizontal dashed line as a reference to the red arrow on the crack. The measured angles of the crack for each axial orientation are presented in population plot as shown in Fig. $4 d-f$. The $<100>$ pillar has four $<110>$ directions on its sidewall with angles of $0^{\circ}, 90^{\circ}, 180^{\circ}$, and $270^{\circ}$, as indicated by the vertical dashed lines. The column data clearly shows four highprobability crack locations at around $45^{\circ}, 135^{\circ}, 225^{\circ}$, and $315^{\circ}$ between $\langle 110\rangle$ directions (d). The $<110>$ pillar has two $<110>$ directions on its sidewall at $55^{\circ}$ and $235^{\circ}$ (vertical dashed lines), and the locations of cracks are clustered around $145^{\circ}$ and $325^{\circ}(\mathrm{e})$. Although obvious peaks of clustered column data for the locations of cracks are not exhibited since there are six $<110\rangle$ directions on its sidewall at $0^{\circ}, 60^{\circ}, 120^{\circ}, 180^{\circ}, 240^{\circ}$, and $300^{\circ}$, most cracks for the $<111>$ pillar are found at $<112>$ directions between the $<110>$ directions, as with other pillars. Hence, despite showing very little anisotropy in expansion, it is evident that lithiation-induced cracks in 
crystalline Ge pillars develop between the $\langle 110\rangle$ directions of preferential lithiation regardless of initial axial pillar orientation and pillar diameter.

In summary, we present the fracture behavior of single crystalline Ge pillars upon electrochemical lithiation. Relatively isotropic expansion of crystalline $\mathrm{Ge}$ upon lithiation enhances fracture resistance while anisotropic expansion of crystalline Si induces concentration of tensile hoop stress on the surface. However, crystalline Ge still shows a small amount of anisotropy in its lithiation-induced expansion due to preferential lithiation along the <110> directions, just as crystalline $\mathrm{Si}$ does. The increase of dimension of Ge pillar along the $<110>$ directions is $10 \%$ larger than along the $\langle 100\rangle$ directions while Si shows 5 times larger increase along the $\langle 110\rangle$ directions compared to the $\langle 100\rangle$ directions. In spite of the small anisotropic expansion, it affects fracture behavior of crystalline Ge. As presented in our previous study on $\mathrm{Si}$, Ge pillars also show favored fracture sites between neighboring <110> directions. We believe that our observation on Ge pillars may provide a better understanding into the volume expansion and fracture behavior. Furthermore, the study of size effect for fracture might provide useful guidelines for preventing the mechanical fracture of Ge anode electrode for high-density Li-ion batteries.

\section{Acknowledgements}

Y.C. acknowledges the support by the US DOE, Office of Basic Energy Sciences, Division of Materials Sciences and Engineering under Contract DE-AC02-76SF00515. W.D.N. and I.R. gratefully acknowledge support from the Office of Science, Office of Basic Energy Sciences, of the U.S. Department of Energy under Contract No. DE-FG02-04ER46163. We thank to Dr. 
Lucas A. Berla for measuring mechanical properties of lithiated germanium by nanoindentation.

\section{Additional Information}

The authors declare no competing financial interests. Supplementary information accompanies this paper online. Correspondence should be addressed to Y.C (yicui@ stanford.edu).

\section{References}

1. Armand, M. \& Tarascon, J.M. Building better batteries. Nature 451, 652-657 (2008).

2. Chan, C.K. et al. High-performance lithium battery anodes using silicon nanowires. Nature nanotechnology 3, 31-35 (2008).

3. Choi, N.-S., Yao, Y., Cui, Y. \& Cho, J. One dimensional Si/Sn - based nanowires and nanotubes for lithium-ion energy storage materials. Journal of Materials Chemistry 21, 9825-9840 (2011).

4. Beaulieu, L.Y., Eberman, K.W., Turner, R.L., Krause, L.J. \& Dahn, J.R. Colossal Reversible Volume Changes in Lithium Alloys. Electrochemical and Solid-State Letters 4, A137A140 (2001).

5. Rhodes, K., Dudney, N., Lara-Curzio, E. \& Daniel, C. Understanding the Degradation of Silicon Electrodes for Lithium-Ion Batteries Using Acoustic Emission. Journal of The Electrochemical Society 157, A1354 (2010).

6. Obrovac, M.N., Christensen, L., Le, D.B. \& Dahn, J.R. Alloy Design for Lithium-Ion Battery Anodes. Journal of The Electrochemical Society 154, A849-A855 (2007).

7. Liu, X.H. \& Huang, J.Y. In situ TEM electrochemistry of anode materials in lithium ion batteries. Energy \& Environmental Science 4, 3844-3860 (2011).

8. McDowell, M.T., Lee, S.W., Nix, W.D. \& Cui, Y. 25th anniversary article: Understanding the lithiation of silicon and other alloying anodes for lithium-ion batteries. Adv Mater 25, 4966-4985 (2013). 
9. Limthongkul, P., Jang, Y.-I., Dudney, N.J. \& Chiang, Y.-M. Electrochemically-driven solid-state amorphization in lithium-silicon alloys and implications for lithium storage. Acta Materialia 51, 1103-1113 (2003).

10. Li, J. \& Dahn, J.R. An In Situ X-Ray Diffraction Study of the Reaction of Li with Crystalline Si. Journal of The Electrochemical Society 154, A156-A161 (2007).

11. Key, B. et al. Real-time NMR investigations of structural changes in silicon electrodes for lithium-ion batteries. Journal of the American Chemical Society 131, 9239-9249 (2009).

12. Ruffo, R., Hong, S.S., Chan, C.K., Huggins, R.A. \& Cui, Y. Impedance Analysis of Silicon Nanowire Lithium Ion Battery Anodes. The Journal of Physical Chemistry C 113, 11390-11398 (2009).

13. Lee, S.W., McDowell, M.T., Choi, J.W. \& Cui, Y. Anomalous shape changes of silicon nanopillars by electrochemical lithiation. Nano letters 11, 3034-3039 (2011).

14. Lee, S.W., Berla, L.A., McDowell, M.T., Nix, W.D. \& Cui, Y. Reaction Front Evolution during Electrochemical Lithiation of Crystalline Silicon Nanopillars. Israel Journal of Chemistry 52, 1118-1123 (2012).

15. Goldman, J.L., Long, B.R., Gewirth, A.A. \& Nuzzo, R.G. Strain Anisotropies and SelfLimiting Capacities in Single-Crystalline 3D Silicon Microstructures: Models for High Energy Density Lithium-Ion Battery Anodes. Advanced Functional Materials 21, 24122422 (2011).

16. Liu, X.H. et al. Anisotropic swelling and fracture of silicon nanowires during lithiation. Nano letters 11, 3312-3318 (2011).

17. Lee, S.W., McDowell, M.T., Berla, L.A., Nix, W.D. \& Cui, Y. Fracture of crystalline silicon nanopillars during electrochemical lithium insertion. Proceedings of the National Academy of Sciences of the United States of America 109, 4080-4085 (2012).

18. Liu, X.H. et al. Size-Dependent Fracture of Silicon Nanoparticles During Lithiation. ACS Nano 6, 1522-1531 (2012).

19. Ryu, I., Lee, S.W., Gao, H., Cui, Y. \& Nix, W.D. Microscopic model for fracture of crystalline Si nanopillars during lithiation. Journal of Power Sources 255, 274-282 (2014).

20. Park, M.H. et al. Germanium nanotubes prepared by using the Kirkendall effect as anodes for high-rate lithium batteries. Angew Chem Int Ed Eng/ 50, 9647-9650 (2011). 
21. Liu, X.H. et al. Reversible nanopore formation in Ge nanowires during lithiationdelithiation cycling: an in situ transmission electron microscopy study. Nano letters 11, 3991-3997 (2011).

22. Park, M.H., Kim, K., Kim, J. \& Cho, J. Flexible dimensional control of high-capacity Liion-battery anodes: from $\mathrm{OD}$ hollow to $3 \mathrm{D}$ porous germanium nanoparticle assemblies. Adv Mater 22, 415-418 (2010).

23. Baggetto, L.c. \& Notten, P.H.L. Lithium-Ion (De)Insertion Reaction of Germanium Thin-Film Electrodes: An Electrochemical and In Situ XRD Study. Journal of The Electrochemical Society 156, A169 (2009).

24. Chang, $C$. et al. Etching submicrometer trenches by using the Bosch process and its application to the fabrication of antireflection structures. Journal of Micromechanics and Microengineering 15, 580-585 (2005).

25. Hsu, C.M., Connor, S.T., Tang, M.X. \& Cui, Y. Wafer-scale silicon nanopillars and nanocones by Langmuir-Blodgett assembly and etching. Applied Physics Letters 93, 133109 (2008).

26. Liang, W. et al. Tough Germanium Nanoparticles under Electrochemical Cycling. ACS Nano 7, 3427-3433 (2013). 